\title{
Etapificación pre-operatoria con TC de pacientes con cáncer mamario. Alternativa a la RM en un hospital público
}

\author{
Dres. Flavia Pizzolon $F^{(1)}$, Eleonora Horvath ${ }^{(2)}$, Heriberto Wenzel $K^{(1)}$, Fernando Cádiz V(1), Gonzalo Serrano B(1), TMs.
} Marcelo Aburto $\boldsymbol{R}^{(1)}$, Oscar Morgado $H^{(1)}$, Juan Baina $D^{(1)}$, María Paz Duran $C^{(2)}$.

1. Servicio de Radiología, Hospital Padre Hurtado. Santiago - Chile.

2. Departamento de Imágenes, Clínica Alemana de Santiago. Facultad de Medicina Clínica Alemana, Universidad del Desarrollo. Santiago - Chile

\section{Preoperative CT staging of breast cancer patients. Alternative to MRI in a public hospital}

\begin{abstract}
The preoperative study in search of multifocality, multicentricity and bilaterality is essential for the proper therapeutic management of breast cancer. The reference standard to accomplish this is breast Magnetic Resonance Imaging (MRI). Based on the literature, we adapted the thorax CT protocol for the study of the breast. The aim of this paper is to demonstrate the feasibility of CT with emphasis on the breast, as an alternative to preoperative MRI in places where it is not available. Pre-operative CT was performed in all patients with newly diagnosed breast cancer between August 2013 and September 2014. The studies were reviewed retrospectively and according to the results, were divided into four categories. Group 1: CT provided no new information. 1A: concordant with conventional screening procedures (previous mammogram (Mx) and ultrasound (US)), 1B: cancer visible on Mx and/or US, but without representation on CT. Group 2: On detection of additional lesions, radiological staging was improved, without changing the type of surgical approach. Group 3: Demonstrated new non-malignant lesions and caused unnecessary surgery. Group 4: Successfully changed the type of surgery planned based on conventional studies. In the 13 months, 47 preoperative mammary CT were performed in 46 patients (ages: from 34 to 81 years, average 56.7 years). The distribution by groups was: Group $1 A(79 \%)$, Group $1 B(8,3 \%)$, Group $2(8.3 \%)$, Group $3(0 \%)$ and Group $4(4.1 \%)$. In $87.5 \%$ of the studies the primary lesion was visualized and its extension could be evaluated. Six cancers were not identified: they corresponded to one case in which no intravenous contrast was administered due to renal failure, a positive negative, where there were only axillary lymphadenopathy of pulmonary origin and four DCIS (8.3\%). In our practice, preoperative CT favorably influenced therapeutic decision-making, improved the radiological staging in the $8.3 \%$, and successfully changed the surgical approach in the $4.1 \%$ of the patients.
\end{abstract}

Keywords: Breast cancer, CT, Staging.

Resumen: El estudio preoperatorio en búsqueda de multifocalidad, multicentricidad y bilateralidad es fundamental para el adecuado manejo terapéutico del cáncer (CA) mamario. El estándar de referencia para realizarlo es la resonancia magnética (RM) mamaria. Basados en la literatura, adaptamos el protocolo de TC de tórax para el estudio de la mama. El objetivo de este trabajo es demostrar la factibilidad de la TC con énfasis en mama como método alternativo a la RM pre-operatoria, en lugares donde ésta no está disponible. Se realizaron TC pre-operatorias en todas las pacientes con diagnóstico reciente de CA mamario entre agosto de 2013 y septiembre de 2014. Los estudios fueron revisados en forma retrospectiva y según su resultado se los dividió en cuatro categorías. Grupo1: La TC no aportó información nueva. 1A: concordante con exámenes convencionales (Mx y US previos), 1B: cáncer visible en Mx y/o US, pero sin representación en la TC. Grupo 2: Al detectar lesiones adicionales, mejoró la etapificación radiológica, sin cambiar el tipo de la cirugía planificada. Grupo 3: Demostró nuevas lesiones no malignas y causó cirugía innecesaria. Grupo 4: Cambió correctamente el tipo de cirugía planeada en base a los estudios convencionales. En los 13 meses se realizaron 47 TC mamarias preoperatorias en 46 pacientes (edades: de 34 a 81 años, promedio: 56,7 años) La distribución por grupos fue: Grupo $1 A$ (79\%), Grupo 1B (8,3\%), Grupo 2 (8,3\%), Grupo $3(0 \%)$ y Grupo 4 (4,1\%). En el 87,5\% de los estudios se visualizó la lesión primaria y se pudo evaluar su extensión. Seis cánceres no fueron identificados: correspondieron a un caso en que no se administró contraste intravenoso por falla renal, un verdadero negativo, en donde sólo existían adenopatías axilares de origen pulmonar y cuatro CDIS (8,3\%). En nuestra práctica, la TC preoperatoria influyó favorablemente en la toma de decisiones terapéuticas, mejoró la etapificación radiológica en el 8,3\% y cambió correctamente el enfoque quirúrgico en el $4,1 \%$ de las pacientes.

Palabras clave: Cancer mamario, Etapificación, Tomografía computada.

Pizzolon F, Horvath E, Wenzel H, Cádiz F, Serrano G, Aburto M et al. Etapificación pre-operatoria con TC de pacientes con cáncer mamario. Alternativa a la RM en un hospital público. Rev Chil Radiol 2014; 20(4): 135-140.

Correspondencia: Flavia Pizzolon F. / flaviapizzolon@hotmail.com

Artículo ganador del primer premio del Congreso Chileno de Radiología 2014. 


\section{Introducción}

De acuerdo a datos de la American Cancer Society el cáncer (CA) de mama es el segundo más frecuentemente diagnosticado entre las mujeres de Estados Unidos y una de cada ocho (12,5\%) desarrollarán CA invasivo a lo largo de la vida(1). Es la segunda causa de muerte por cáncer y se estima que su tasa de mortalidad está descendiendo desde 1989, lo que se cree, estaría determinado por la detección temprana y por las mejoras terapéuticas ${ }^{(1)}$.

El tratamiento y pronóstico del CA mamario están determinados predominantemente por el estadío de la enfermedad. Una vez realizado el diagnóstico, la información obtenida de la etapificación ayudará a elegir entre una terapia conservadora y mastectomía, quimioterapia pre o postoperatoria, biopsia de ganglio centinela 0 disección axilar y uso de radioterapia ${ }^{(1)}$.

En la etapificación loco-regional el estándar de referencia es la resonancia magnética $(\mathrm{RM})$ mamaria con gadolinio intravenoso, técnica validada más sensible y específica para la caracterización de extensión tumoral, multicentricidad, multifocalidad y bilateralidad ${ }^{(2,3)}$. Tanto la RM como la tomografía computada (TC) dinámicas se fundamentan en su capacidad para demostrar la neo-angiogénesis tumoral, vascularización anómala que caracteriza los tumores sólidos ${ }^{(4)}$.

Sin embargo, algunos pacientes no pueden someterse a una RM debido a contraindicaciones (clips metálicos, marcapasos, etc.) o a condiciones limitantes, por ejemplo claustrofobia $^{(5)}$. Entre las opciones alternativas a la RM se encuentran varias modalidades sofisticadas, como el PEM (mamografía por emisión de positrones ${ }^{(6)}$, RM dedicada de mama y TC dedicada de mama (esta aún en etapa de desarrollo clínico) ${ }^{(7)}$ y la angio-mamografía digital o mamografía contrastada (realizada en dos adquisiciones, con y sin administración de contraste i.v. y sustracción de las imágenes obtenidas)(8).

En este trabajo planteamos la utilización de la TC multidetector convencional con énfasis en mama, como alternativa disponible y de bajo costo en la etapificación loco-regional del CA mamario en caso de no tener disponibilidad de RM.

\section{Objetivos}

Demostrar la utilidad de la TC convencional adaptada al estudio de la mama como método alternativo a la RM preoperatoria en el Hospital Padre Hurtado (HPH).

\section{Material y método}

Se incluyeron en esta revisión 46 mujeres consecutivas con CA mamario diagnosticado entre agosto de 2013 y septiembre de 2014 en el Hospital Padre Hurtado. Las pacientes contaban con estudios convencionales, mamografía (Mx) y ecografía (US) y con biopsias percutáneas (biopsia Core y/o estereotáxia) que permitieron el diagnóstico.

Al momento de realizar la TC pre-operatoria de etapificación de rutina, el protocolo estándar de tórax fue sustituido por el de la TC con énfasis en mama. Todas las pacientes firmaron consentimiento informado.

\section{Técnica de estudio}

Se utilizó un tomógrafo multidetector de Toshiba Aquilion de 16 canales. En base a publicaciones previas ${ }^{(5,9)}$ se diseñó un protocolo especial de TC de tórax multi-detector de baja dosis con énfasis en mama, en posición de decúbito prono. Estudio trifásico: sin contraste, contraste yodado $100 \mathrm{cc}$, a los 90 seg. y $300 \mathrm{seg}$. (2-3 $\mathrm{cc} / \mathrm{seg}$ ). Baja dosis de radiación: 120 Kvp y 50-100 mAs, dependiendo del tamaño de las mamas. FOV de $34 \mathrm{~cm}$. Espesor de corte: $3 \mathrm{~mm}$. Colimación: $1 \mathrm{~mm}$. Barrido: desde la base del cuello hasta las bases pulmonares en apnea. Fase tardía sólo enfocada en la región mamaria. Post-proceso: MIP sagital y coronal de data de volumen de $1 \mathrm{~mm}$, filtro de reconstrucción, Kernel B30.

Si bien el estudio puede ser realizado en decúbito supino, para lograr un posicionamiento similar al de las imágenes de RM, se fabricó, un soporte para poder realizarlo en decúbito prono (Figuras 1 y 2). Se trata de una base radio-transparente de plumavit, de forma rectangular con un orificio de forma rectangular, donde se posicionan las mamas. Este se ubica sobre la mesa del tomógrafo y la paciente se recuesta sobre él. Consta de dos partes, una superior, para colocar la cabeza de la paciente y una parte inferior para el cuerpo, que gradualmente disminuye de espesor para dar comodidad al abdomen. La apertura donde se posicionan las mamas mide $42 \mathrm{~cm}$ por $20 \mathrm{~cm}$, sus bordes son romos para evitar molestias y compresión de estructuras vasculares en axilas ${ }^{(9)}$.

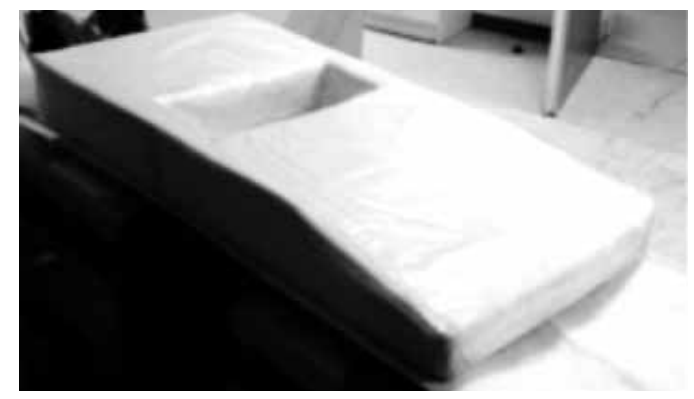

Figura 1. Soporte para posicionamiento diseñado en plumavit, recubierto de material impermeable y lavable.

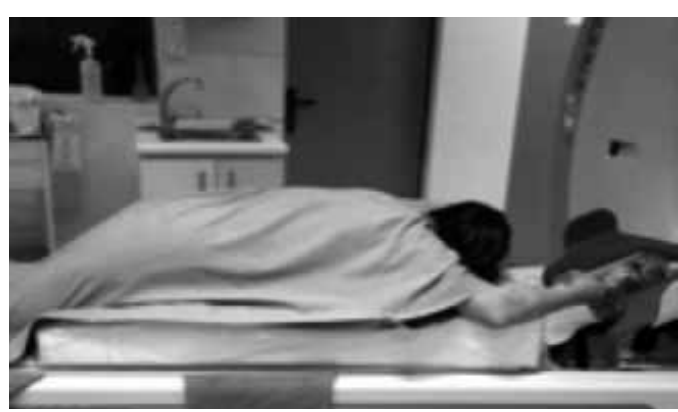

Figura 2. Paciente ubicada en el tomógrafo en decúbito prono, recostada sobre el soporte. 


\section{Interpretación de los estudios}

Dos radiólogos especializados en imágenes mamarias (FP y HW) revisaron las mamografías, ecografías y realizaron las biopsias ya sea Core o estereotaxia, de las pacientes. Uno de ellos (FP) evaluó las imágenes mamarias de la TC y los radiólogos de cuerpo del Servicio de Imágenes de HPH se encargaron del informe del resto del estudio.

Conociendo los resultados del escáner, se discutió caso a caso a los eventuales cambios en la terapéutica con los cirujanos de mama del Hospital.

Se creó una base de datos en Excel (versión Office: Mac 2011) para consignar diferentes parámetros, como: representación o no del cáncer en la TC, tamaño tumoral en las tres modalidades de imagen, concordancia de la TC con exámenes convencionales, detección de nuevas lesiones en las mamas, iagnóstico de lesiones extramamarias, detectabilidad de linfonodos patológicos, necesidad de ecografía de second look y complicaciones durante la realización de los estudios, entre otros.

En cuanto a los ganglios axilares, se definieron características de sospecha, como hipercaptación de contraste, aumento de tamaño, bordes irregulares y pérdida del hilio adiposo. Para cada caso se definió un CA índice diagnosticado por los exámenes convencionales (ej. un cáncer conocido o adenopatías axilares) y dos en caso de bilateralidad conocida. Una vez recibido el resultado anatomopatológico de la pieza operatoria, se evaluó el rendimiento de la TC según la clasificación utilizada para valorar la RM pre-operatoria ${ }^{(10)}$ en las siguientes cuatro categorías:

Grupo 1: La TC no aportó información nueva en cuanto a extensión tumoral en las mamas.

- 1A: concordante con exámenes convencionales (Mx y US previos) (Figura 3).

- 1B: cáncer visible en Mx y/o US, pero sin representación en la TC.

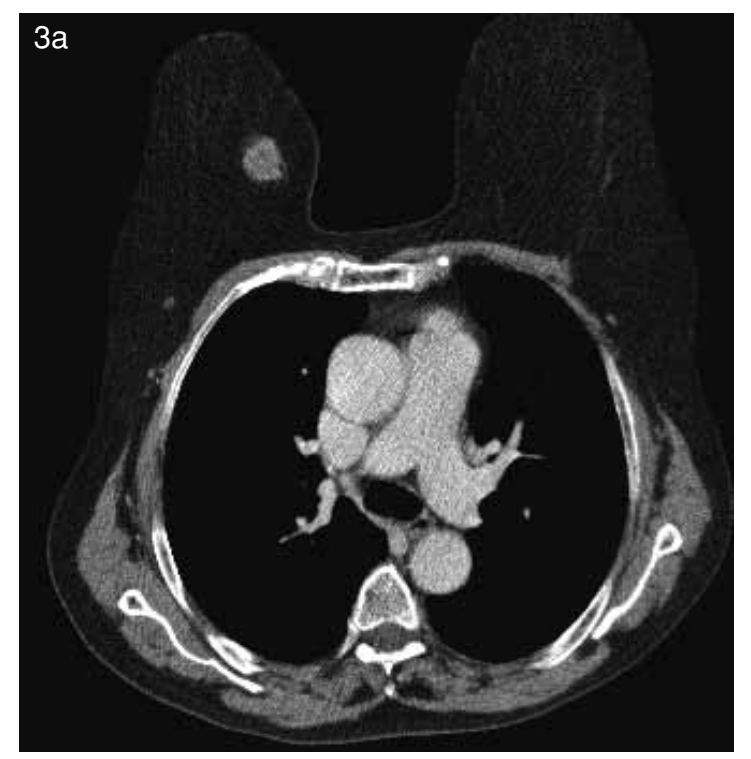

Grupo 2: La TC detectó lesiones adicionales y mejoró la etapificación radiológica previa, pero no cambió el enfoque quirúrgico predeterminado.

Grupo 3: Al demostrar nuevas lesiones no malignas, la TC causó cirugía adicional inútilmente.

Grupo 4: Gracias a los datos proporcionados, la TC generó un cambio acertado en el tratamiento quirúrgico (Figura 4).

\section{Resultados}

En el periodo de 13 meses se realizaron 47 estudios preperatorios con TC en 46 mujeres de entre 34 y 81 años, mediana 60 años. Una paciente tuvo dos exámenes por control de quimioterapia neo-adyuvante. Tres pacientes tenían CA bilateral, dos de ellos diagnosticados previamente, el tercero corresponde a TC-only. En total se estudiaron 48 lesiones índice conocidos, su tamaño varió de 6 $\mathrm{mm}$ a $100 \mathrm{~mm}$ (mediana de $26 \mathrm{~mm}$ ).

Según su tipo histológico correspondieron a $36 \mathrm{CDI}(75 \%), 4 \mathrm{CDIS}(8,3 \%), 5 \mathrm{CLI}(10,4 \%), 1 \mathrm{CA}$ medular $(2,0 \%), 1 \mathrm{CA}$ mucinoso $(2,0 \%)$ y 1 caso con adenopatía metastásica $(2,0 \%)$ de origen extramamario (pulmonar) (Figura 5).

De las 48 lesiones estudiadas se logró identificar $42(87,5 \%)$ en TC, las $6(12,5 \%)$ lesiones no visualizadas correspondieron a un caso en que no se administró contraste i.v. por falla renal, a un verdadero negativo en donde sólo existían adenopatías axilares de origen pulmonar y a cuatro CDIS, representados por microcalcificaciones agrupadas ( 3 casos) y lesión papilar con CDIS (1 caso).

Con la administración de contraste i.v. se logró visualizar el $100 \%$ de las lesiones infiltrantes $(n=43)$.

Tres de los casos estudiados correspondieron a recidivas locales $(6,2 \%)$. En un caso se demostró compromiso secundario óseo $(2,0 \%)$.

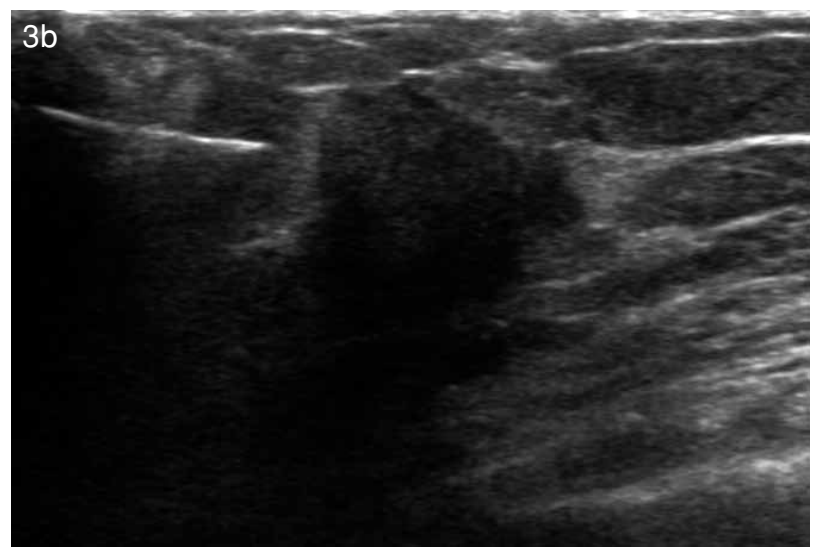

Figura 3. Caso grupo 1. La TC no aporto información nueva a) TC con ventana mediastínica y contraste i.v. muestra nódulo único, hipercaptante, de contornos irregulares. b) US: Biopsia Core del nódulo sospechoso con resultado de CA ductal infiltrante. 

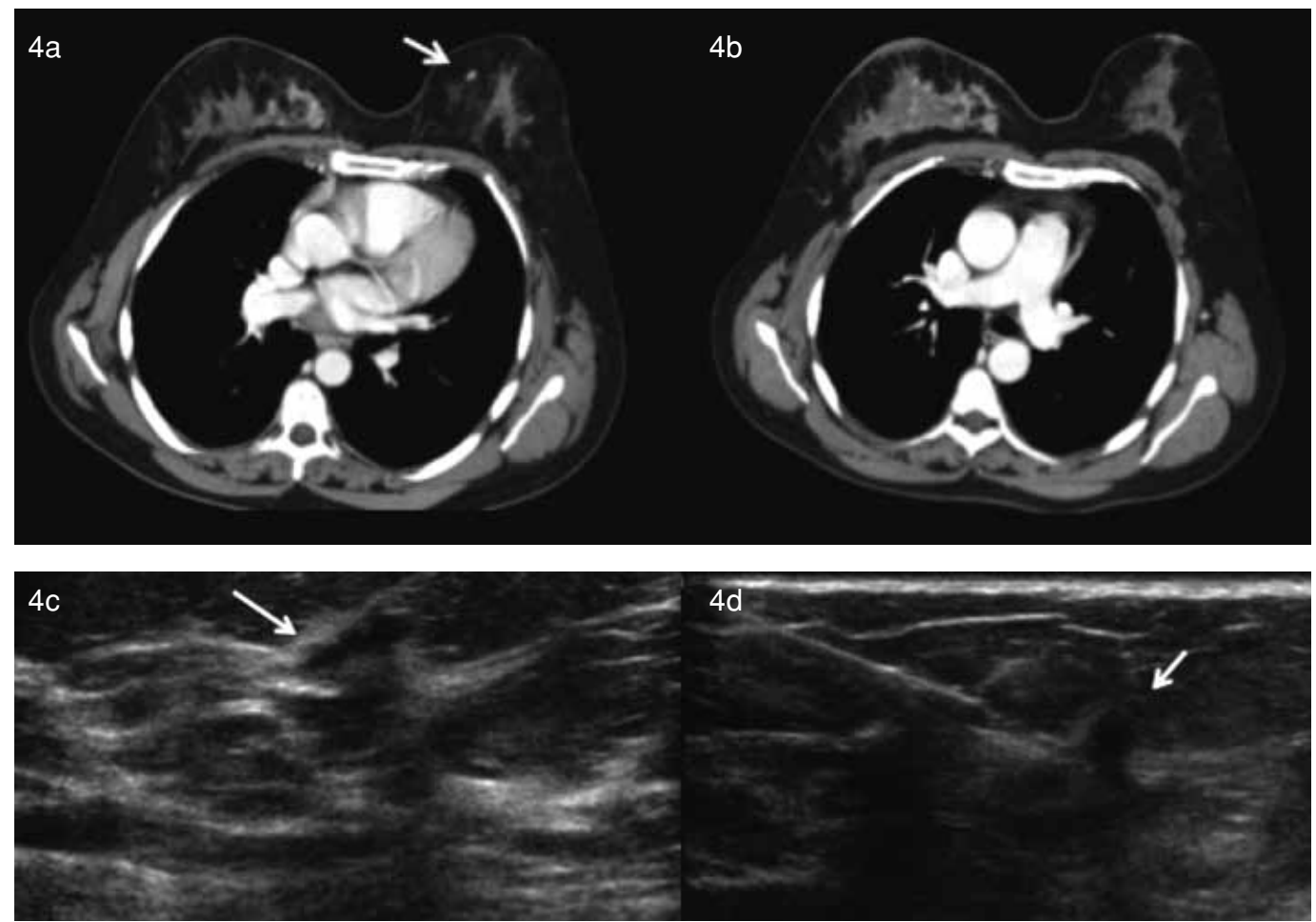

Figura 4. Caso grupo 4. La TC cambió correctamente el tipo de cirugía planeada en base a los estudios convencionales. a, b) TC con ventana mediastínica y contraste i.v. muestra múltiples lesiones hipercaptantes en la mama derecha, conocidas (CA multifocal). El pequeño foco único de la mama izquierda (flecha) fue detectado exclusivamente con TC. c) Ecografía dirigida de "second look" en donde se logra identificar la lesión sospechosa en la mama izquierda. d) Se realizó su biopsia Core bajo US, confirmando otro CA ductal infiltrante (CA bilateral).

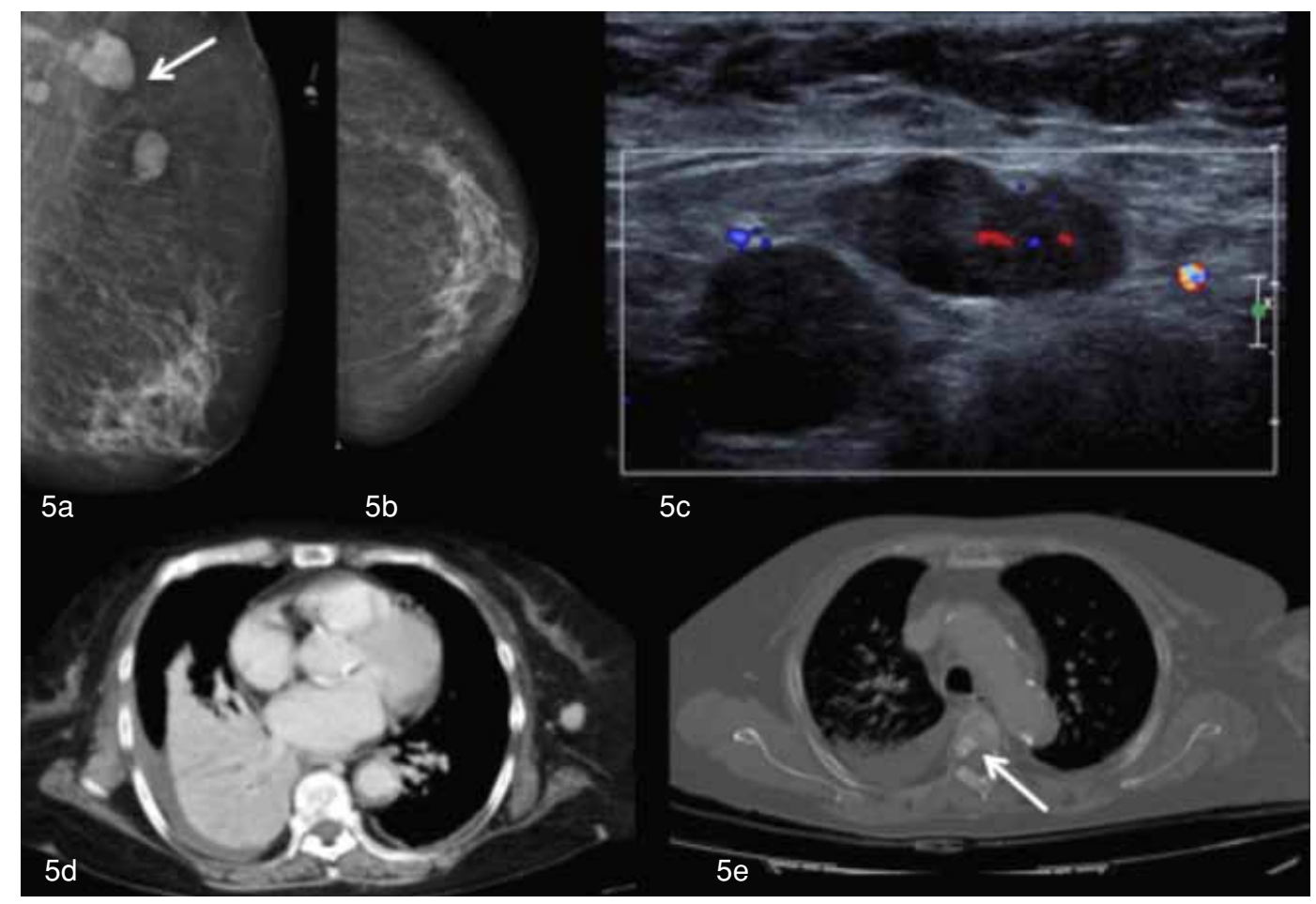

Figura 5. a,b) Mamografía MLO y proyección CC: adenopatías axilares, sin alteraciones parenquimatosas sospechosas. c) Ecografía axilar izquierda: adenopatías sugerentes de metástasis. d) TC, ventana mediastínica: sin captación anormal en el parénquima mamario, sin embargo se evidencia condensación pulmonar y derrame pleural, secundarios a CA pulmonar con adenopatía axilar izquierda. e) TC, ventana ósea: metástasis ósea en cuerpo vertebral. La TC confirmó un caso verdaderamente negativo para CA de mama y, adicionalmente, permitió demostrar la causa de las adenopatías axilares al evaluar simultáneamente el tórax y esqueleto. 


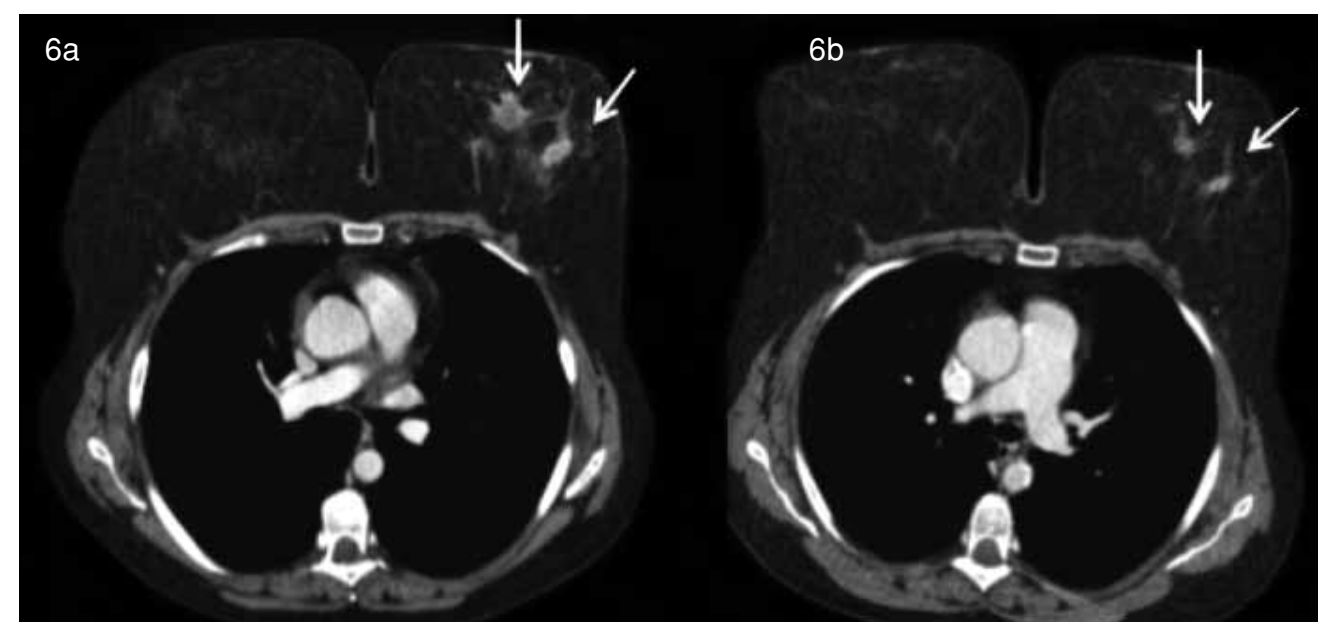

Figura 6. Monitoreo in vivo de respuesta a la QTNA, como una indicación posible para la TC con énfasis en mama: a) TC con ventana mediastínica previo a inicio de QTNA, se visualiza carcinoma ductal infiltrante bifocal en mama izquierda. b) Luego de tres ciclos de QTNA se observa reducción de tamaño de ambas lesiones.

La evaluación del rendimiento de la TC según las cuatro categorías fue la siguiente:

Grupo $11 A:(n=38,79 \%), 1 B(n=4,8,3 \%)$,

Grupo $2(n=4,8,3 \%)$,

Grupo $3(n=0,0 \%)$

Grupo $4(n=2,4,1 \%)$.

En todos los casos se pudo analizar adecuadamente los ganglios axilares. Se evaluaron 43 pacientes con CA infiltrante (no se incluyeron los CDIS), 26 fueron interpretadas con regiones axilares normales, 17 como metastásicas. Hubo dos falsos negativos (un caso de micrometástasis y otro de metástasis de CA medular). Cabe destacar además dos casos de falsos positivos (histiocitosis sinusal y cambios reactivos).

No se registraron complicaciones en relación a la realización de los 47 estudios mamarios con TC.

\section{Discusión}

La etapificación pre-operatoria del CA de mama es fundamental para definir un plan terapéutico óptimo y de esta manera mejorar el pronóstico a las pacientes.

El método recomendado es la $\mathrm{RM}$, debido a su demostrada capacidad de cambiar adecuadamente la conducta terapéutica en el 15 a $20 \%$ de los casos $^{(10-12)}$. Sus ventajas son que no irradia y permite evaluar simultáneamente ambas mamas en secuencias dinámicas y anatómicas, junto con las partes blandas adyacentes, incluyendo linfonodos axilares y mamarios internos. Su desventaja es que no estudia huesos ni pulmones, sitios frecuentes de metástasis, es de alto costo, es de disponibilidad restringida, de realización lenta y tiene contraindicaciones como implantes metálicos, marcapasos, claustrofobia, etc.

La alternativa que planteamos es la TC convencional con énfasis en mama en decúbito prono, ya que es de fácil acceso, se encuentra disponible en el sistema de atención pública y es de bajo costo. Permite una adecuada visualización de las mamas, axilas, huesos y pulmones, representando así un método de etapificación doble, local y a distancia. Su realización toma pocos minutos. Al adquirir las imágenes en decúbito prono el parénquima mamario se despliega, evitando compresiones que puedan alterar la impregnación con medio de contraste y se evitan superposición de estructuras ${ }^{(13,14)}$.

Su gran desventaja es el uso de radiación ionizante. Respecto de la radiación recibida por las pacientes, es sabido que exposiciones mayores o iguales a $1 \mathrm{~Sv}$ a temprana edad incrementan el riego de desarrollar cáncer mamario. Los efectos de radiaciones de bajo nivel ( $\leq 10$ cGy) están menos definidos ${ }^{(15)}$. La tomografía dedicada a la mama con parámetros de 120 Kvp y 100 mAs para una sola adquisición en apnea significa una dosis recibida de 3,3 mSv. En total suma 9,9 mSv para el estudio dinámico. A modo de comparación, una mamografía bilateral significa 4,7 a 3,7 mSv, dependiendo de si es analógica o digital(16). Por lo tanto, con el protocolo de estudio propuesto, podemos minimizar la dosis de radiación y conseguir una valiosa información adicional en preoperatorio.

Otra desventaja es la posibilidad de reacciones adversas, puesto que esta técnica requiere de la administración de contraste iodado intravenoso para poder visualizar las lesiones malignas a través de la demostración de la neo-angiogénesis tumoral, principio en el cual también se fundamenta la RM contrastada. En uno de nuestros casos hubo falla renal por lo cual no se administró contraste iodado y no se logró demostrar un cáncer mamario ya conocido. Hasta la fecha no tuvimos complicaciones alérgicas ni inconvenientes al inyectar el medio de contraste.

En general, hemos encontrado similitud al comparar los hallazgos en TC con los hallazgos mamográficos y ecográficos, gracias a los cortes finos de $1 \mathrm{~mm}$, permitiendo detectar incluso un cáncer de $6 \mathrm{~mm}$. En nuestra experiencia preliminar, la TC aportó más información sobre el estatus ganglionar que la Mx y US juntas.

Las lesiones nuevas encontradas exclusivamente en la TC (TC-only) correspondieron a los dos casos del 
Grupo 4. Ambas pacientes se sometieron a una nueva ecografía dirigida (US second-look), lográndose identificar en una de ellas un nódulo en un cuadrante diferente y en la segunda un nódulo contralateral.

En esta serie preliminar por el momento sólo tenemos un $4.1 \%$ de cambio de conducta quirúrgica (Grupo 4) que parece significativamente menor que lo obtenido en series publicadas de RM pre-operatoria ${ }^{(10-12)}$. No obstante, esto sumado al $8.3 \%$ del Grupo 2 (la TC detectó lesiones adicionales y mejoró la etapificación radiológica previa, sin cambio el enfoque quirúrgico predeterminado) significa que en nuestro hospital ahora el $12,4 \%$ de los pacientes tienen acceso a una mejor etapificación pre-operatoria, lo que supone un mejor tratamiento y probablemente una menor tasa de recurrencia local en el futuro.

En este trabajo evaluamos el rendimiento de la TC pre-operatoria. Intuimos que podría tener otras indicaciones similares a las de la RM mamaria. Puede servir por ejemplo en el monitoreo de quimioterapia neoadyuvante (QTNA): una de nuestras pacientes tuvo una segunda exploración por QTNA y el estudio proporcionó información similar al de la RM en cuanto al tamaño de la lesión índice y compromiso linfático regional (Figura 6). Puede ser útil también en confirmar o descartar una recidiva, de hecho, tres de las 46 pacientes se estudiaron por recidivas locales, en donde fue notoria la presencia de lesiones hipercaptantes sospechosas y el compromiso de estructuras vecinas.

Durante el período de estudio se realizó una TC de una paciente con CA mamario tratado hace más de diez años, quien presentó hallazgos mamográficos de sospecha de recidiva en la zona de la cicatriz. En este caso la realización de la RM estaba contraindicada debido a la presencia de clips cerebrales por tratamiento de aneurisma. La TC con énfasis en mama visualizó los clips metálicos del lecho quirúrgico y la cicatriz fibrosa, sin realce tras la administración de contraste i.v. La paciente continúa en seguimiento hasta la actualidad.

Hemos visto que la técnica, fuera de sus desventajas ya mencionadas, tiene limitaciones para detectar los canceres in situ. En nuestra serie ninguno de los 4 CDIS tenía representación en la TC. Cabe destacar que tres de ellos fueron de tamaño pequeño y mamográficamente aparecieron como tenues microcalcificaciones agrupadas y el cuarto correspondía a un papiloma de $19 \times 3 \mathrm{~mm}$, asociado a CDIS. No tuvimos aún la oportunidad de evaluar CDIS extensos con o sin microinvasión, que tal vez, al igual que en la RM, podrían tener traducción en la TC dada un angiogénesis tumoral más importante.

\section{Conclusión}

Si bien nuestro estudio cuenta con un número acotado de casos y no pudo compararse con el estándar de oro (RM), ya que no se encuentra disponible, en nuestra práctica pudimos observar cambios favorables en la etapificación radiológica y en la conducta terapéutica, que plantean la TC multidetector con énfasis en mama, como alternativa útil a la $\mathrm{RM}$ mamaria pre-operatoria en lugares donde ésta no se encuentra disponible.

\section{Bibliografía}

1. Lee S, Jain P, Jethwa S, Triphaty D, Yamashita M. Radiologists Role in Breast Cancer Staging: Providing Key Information for Clinicians. Radiographics 2014; 34: 330-342.

2. Fischer U, Kopka L, Grabbe E. Breast Carcinoma: Effect of Preoperative Contrast-enhanced MR Imaging on the Therapeutic Approach. Radiology 1999; 213(3): 881-888.

3. Sardanelli, F, Giuseppetti, GM, Panizza P et al. Sensitivity of MRI versus mammography for detecting foci of multifocal, multicentric breast cancer in fatty and dense breasts using the whole-breast pathologic examination as a gold standard. AJR Am J Roentgenol 2004; 183: 1149-1157.

4. Cuenod CA, Fournier L, Balvay D, Guinebretière JM. Tumor angiogenesis: pathophysiology and implications for contrast-enhanced MRI and CT assessment. Abdom Imaging 2006; 31: 188-193.

5. Perrone A, Lo Mele L, Sassi S, Marini M, Testaverde L, Izzo L et al. MDCT of the Breast. AJR Am J Roentgenol 2008; 190: 1644-1651.

6. Berg WA, Madsen KS, Schilling K, Tartar M, Pisano ED, Larsen LH et al. Comparative Effectiveness of Positron Emission Mammography and MRI in the Contralateral Breast of Women With Newly Diagnosed Breast Cancer. AJR Am J Roentgenol 2012; 198(1): 219-232.

7. O'Connell A, Conover DL, Zhang Y, Seifert P, Logan-Young W, Lin CF et al. Cone-beam CT for breast imaging: Radiation dose, breast coverage, and image quality. AJR Am J Roentgenol 2010; 195: 496-509.

8. Dromain C, Balleyguier C, Muller S, Mathieu MC, Rochard F, Opolon $\mathrm{P}$ et al. Evaluation of tumor angiogenesis of breast carcinoma using contrast enhanced digital mammography. Am J Roentgenol 2006; 187: W528-W537.

9. Lee WJ, Seo BK, Cho PK, Yie A, Cho KR, Woo OH et al. The Clinical Use of Low-Dose Multidetector Row Computed Tomography for Breast Cancer Patients in the Prone Position. J Breast Cancer 2010; 13(4): 357-365.

10. Horvath E, Galleguillos M, Maldonado P, Fernández M, Sañudo M, Tiscornia $P$ et al. Resonancia magnética mamaria preoperatoria: la gran polémica. Rev Chil Radiol 2011; 17(4): 166-173.

11. Houssami N, Ciatto S, Macaskill P et al. Accuracy and surgical impact of magnetic resonance imaging in breast cancer staging: Systematic review and meta-analysis in detection of multifocal and multicentric cancer. J Clin Oncol 2008; 26(19): 3248-3258.

12. Plana MN, Carreira C, Muriel A et al. Magnetic resonance imaging in the preoperative assesment of patients with primary breast cancer: systematic review of diagnostic accuracy and meta-analysis. Eur Radiol 2012; 22(1): 26-38.

13. Prionas ND, Lindfors KK, Ray S, Huang SY, Beckett LA et al. Contrast-enhanced Dedicated Breast CT: Initial Clinical Experience. Radiology 2010; 256(3): 714-723.

14. Lindfors KK, Boone JM, Nelson TR, Yang K, Kwan AL, Miller DF. Dedicated Breast CT: Initial Clinical Experience. Radiology 2008; 246(3): 725-733.

15. Hurwitz LM, Yoshizumi TT, Reiman RE, Paulson EK, Frush DP, Nguyen GT, Toncheva Gl, Goodman PC. Radiation Dose to the Female Breast from 16-MDCT Body Protocols. AJR Am J Roentgenol 2006; 186: 1718-1722.

16. Yaffe MJ, Mainprize JG. Risk of Radiation-induced Breast Cancer from Mammographic Screening. Radiology 2011; 258(1): 98-105. 